\title{
Star trackers for attitude determination
}

\author{
Liebe, Carl Christian
}

Published in:

I E E E Aerospace and Electronic Systems Magazine

Link to article, DOI:

$10.1109 / 62.387971$

Publication date:

1995

\section{Document Version}

Publisher's PDF, also known as Version of record

Link back to DTU Orbit

Citation (APA):

Liebe, C. C. (1995). Star trackers for attitude determination. I E E E Aerospace and Electronic Systems Magazine, 10(6), 10-16. https://doi.org/10.1109/62.387971

\section{General rights}

Copyright and moral rights for the publications made accessible in the public portal are retained by the authors and/or other copyright owners and it is a condition of accessing publications that users recognise and abide by the legal requirements associated with these rights.

- Users may download and print one copy of any publication from the public portal for the purpose of private study or research.

- You may not further distribute the material or use it for any profit-making activity or commercial gain

- You may freely distribute the URL identifying the publication in the public portal

If you believe that this document breaches copyright please contact us providing details, and we will remove access to the work immediately and investigate your claim 


\title{
Star Trackers
}

\section{for Attitude Determination}

\author{
Dr. Carl Christian Liebe \\ Department of Electrophysics, Technical University of Denmark
}

\begin{abstract}
One problem comes to all spacecrafts using vector information. That is the problem of determining the attitude. This paper describes how the area of attitude determination instruments has evolved from simple pointing devices into the latest technology, which determines the attitude by utilizing a CCD camera and a powerful microcomputer. The instruments are called star trackers and they are capable of determining the attitude with an accuracy better than 1 arcsecond. The concept of the star tracker is explained. The obtainable accuracy is calculated, the numbers of stars to be included in the star catalogue are discussed and the acquisition of the initial attitude is explained. Finally the commercial market for star trackers is discussed.
\end{abstract}

\section{INTRODUCTION}

The meaning of the word attitude (or orientation) in spacecraft terminology is essentially "what is up and down." However, the spacecraft is in the space and therefore the

Author's Current Address:

Department of Electrophysics, Technical University of Denmark, 2800 Lyngby, Denmark Manuscript recveived November 2, 1994, revised March 14, 1995.

$0885-8985 / 95 / \$ 4.00$ @ 1995 IEEE words "up and down" lose their meaning. Alternatively, the attitude is determined as the angular departure from some reference. The attitude information is fundamental information for a spacecraft and is typically used to point the solar arrays toward the sun, point the high gain antenna toward the earth after deployment or acquisition of an attitude for a science payload.

The increasing precision of instruments has stressed the demand for a very accurate attitude system during the operational stage. This implies that new attitude determination systems (ADS) must be able to comply with demands on high update rates and accuracies better than 1 arcsecond. Also the trend toward microsatellites has pushed the technology towards lighter instruments and less power consumption for ADSs. Spacecraft ADSs are probably the one spacecraft instrument which has been evolving the most recently. This is due to the CCD technology and the availability of low power microcomputers for space applications.

\section{TRADITIONALATTITUDE DETERMINATION SYSTEMS}

In the past a variety of different ADSs have been used. A traditional ADS consisted of an absolute attitude reference sensor and an inertial sensor. The absolute attitude sensor determined the absolute pointing direction (PD) of the 
spacecraft at regular intervals. This measurement was used to calibrate the inertial sensor, which measured the changes in the attitude between the absolute calibrations.

The following technologies have been utilized for absolute attitude sensors:

- Magnetometers measure the size and orientation of a magnetic field. This strategy requires detailed knowledge of the magnetic field. Accuracies of 1 arcminute are obtainable. The precision depends on the geomagnetic model.

- Radio frequency beacons can be used as a reference of pointing. However if high accuracies are desired, a directional antenna is required. Accuracies of 1 arcminute are obtainable. Because only one reference is utilized the method is only able to determine a Pointing Direction (PD).

- Horizon sensors detect the limp of the earth, typically detecting infrared radiation. Accuracies of approximately 5 arcminutes are obtainable depending on the orbit.

- Sun sensors can be constructed in numerous ways. They all utilize that the sun is the brightest object on the firmament. Constructions vary from detecting whether obtainable. If more than one star is tracked all three angles in the attitude is determined.

The following technologies have been utilized as inertial sensors:

- Gyroscopes are spinning flywheels. If the orientation of the gyroscope is changed, the flywheel will apply a torque on the axis, which is proportional to the angular velocity of the gyroscope. High precision gyroscopes are very expensive. They also suffer from wear out and high drift rates.

- Optical gyroscopes have the advantage of no moving parts. They consist of a coil of double optical fibres. The difference in light propagation in each direction of the optical fiber is proportional to the angular velocity of the optical gyroscope.

- Orbit models are mathematical models of the spacecraft motion. It simulates the motion of the spacecraft, which allows to extrapolate the attitude to a given time.

High quality inertial sensors have a drift rate lower than $0.01 \%$. Below is a table comparing the different absolute attitude sensors:

\begin{tabular}{||l|l|l|l||}
\hline Type & $\begin{array}{l}\text { Initial attitude } \\
\text { acquisition }\end{array}$ & $\begin{array}{l}\text { Degrees of freedom } \\
\text { in attitude }\end{array}$ & Accuracy \\
\hline Magnetometer & Yes & 3 & 1 arcminute \\
\hline $\begin{array}{l}\text { Radio frequency } \\
\text { beacon }\end{array}$ & Yes & 2 & 1 arcminute \\
\hline Horizon sensor & Yes & 2 & 5 arcminutes \\
\hline Sun sensor & Yes & 2 & 1 arcminute \\
\hline Solar panel & Yes & 2 & $1^{\circ}$ \\
\hline Star tracker & No & 3 & 1 arcsecond \\
\hline
\end{tabular}

the sun is present in a cone to high precision instruments that determine the orientation better than 1 arcminute. Because only one reference object is utilized the sun sensor only determines the PD toward the sun.

- Solar panels can also be used as sun sensors. The currents from the different solar panels are monitored. Accuracies of $1^{\circ}$ are obtainable. As the sun is the only reference used, only the $\mathrm{PD}$ toward the sun can be determined.

- Star trackers are beyond dispute the most accurate reference for pointing, because they utilize the fixed stars. The disadvantage of traditional star trackers was that they had to be externally "locked" onto a known star(s). Accuracies better than 1 arcsecond are

\section{STAR TRACKERS}

The rest of this paper will deal with star trackers exclusively. The star trackers described above are first generation star trackers. The present second generation star trackers, also called star imagers, have the advantage compared to first generation star trackers that there is no need for an additional attitude instrument to initially "lock" the star tracker onto a known star(s). The star tracker performs this task by a pattern recognition of the star constellations in the field of view (FOV) and by means of a star catalogue covering the entire firmament. Also no inertial attitude instrument is required as the instrument is equipped with a powerful microcomputer that includes a mathematical model of the spacecraft motion, accurate enough to extrapolate the attitude. 


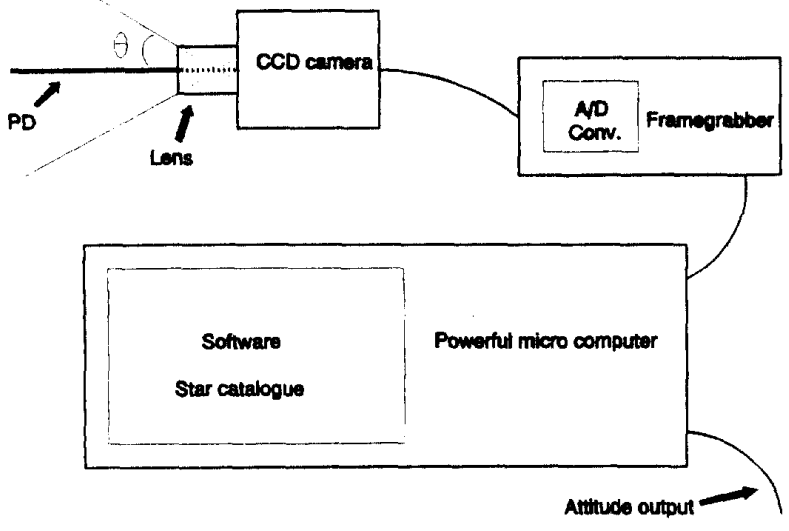

Fig. 1. Principle Diagram of a Second Generation Star Tracker. The Analog CCD Signal is Converted to a Digital Image in the Framegrabber. The Digital Image is Transformed into the Attitude Information by the Computer.

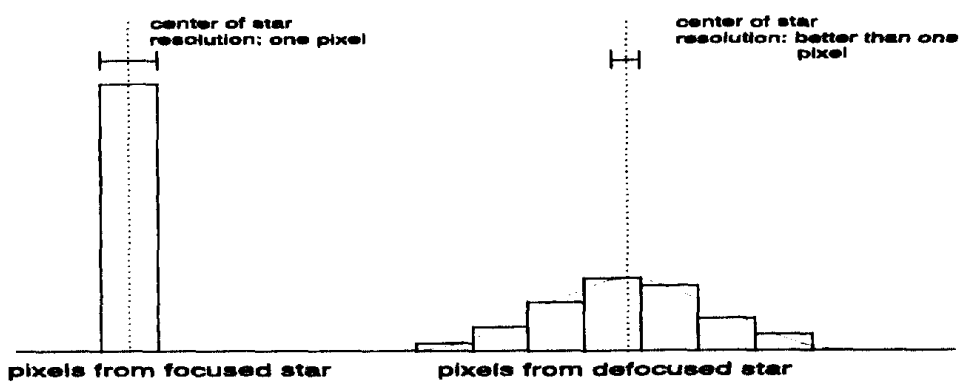

Fig. 3. The Hyperacuity Technique

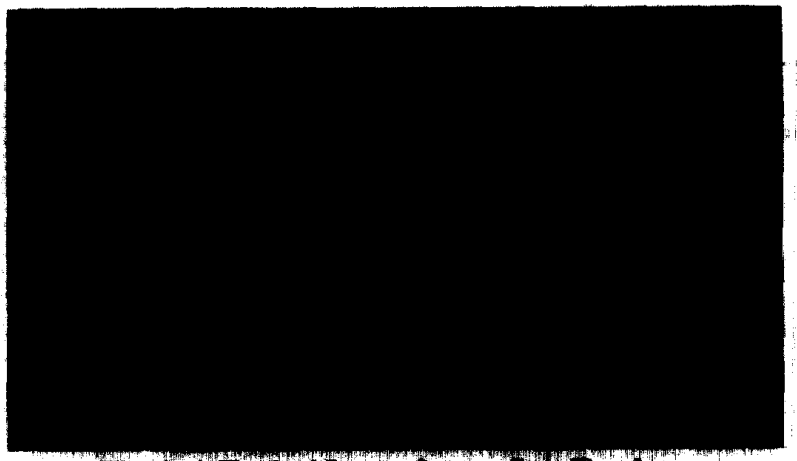

Fg. 2. A Typical lmage from a Stur Tracker

Figure 2 pictures a typical image acquired from a CCD star tracker. The star tracker performs a pattern recognition of the star constellations in the image. In this case the star tracker will identify the stars as a part of the Big Dipper. Based on the celestial coordinates of the identified stars, it is possible to calculate the celestial coordinates of the center of the image and the rotation around it. The center of the image is the same as the PD of the camera. With the PD and with the knowledge of how the camera is mounted on the spacecraft body, it is possible to calculate the attitude of the spacecraft.

A main incentive to choose the star tracker as attitude determinator is the high accuracy. Therefore, the obtainable accuracies of the star tracker are discussed. The angular resolution (in one direction) of one pixel, $\xi$, on the CCD chip is:

$$
\zeta=\frac{2 \theta}{N_{C C D}}
$$

where $\theta$ is the half opening angle of the lens and $N_{C C D}$ is the number of pixels across the CCD chip. A CCD chip typically consists of 300-1,000 pixels in each direction, and $\theta$ is typically $1^{\circ}-20^{\circ}$. It is evident that the desired accuracy cannot be obtained directly. Luckily there are three ways to increase the accuracy of the ADS:

1) Subpixel precision, i.e. to determine the position of a star more accurate than one pixel.

2) The star image includes many stars as seen in Fig. 2. All the 
authors claim to achieve subpixel precision better than $1 / 100$ pixel, others in the order of $1 / 10$ pixel, all dependent on the $\mathrm{S} / \mathrm{N}$ ratio.

As opposed to fancy mathematical models, the algorithm normally utilizes empirical calibrations. Experiments have shown that calibrations are very consistent, even after many years of storage.

\section{Utilizing Multiple Stars In The Image}

An image acquired with a star tracker includes many stars (see Fig. 2). The attitude should be determined on basis of all the stars in the image. If the hyperacuity technique can determine a star position with an accuracy of $e$ Star, the accuracy of the PD $\left(e_{\mathrm{pd}}\right)$ is:

$$
\mathrm{e}_{p d}=\frac{\mathrm{e}_{\text {star }}}{\sqrt{\overline{N_{F O V}}}}
$$

where NFOV is the number of stars in the field of view.

The same phenomenon can be utilized to increase accuracy of the rotation around the PD. This is more complicated, because the accuracy of the rotation is a function of the distance of the individual stars to the optical center. However numerical simulations show that the rotation around the PD is typically determined 4-20 times poorer than the accuracy of the PD itself.

An example: The attitude of a microsatellite is determined with a 2 nd generation star tracker. Across the CCD chip is 650 pixels. The FOV is circular and $22^{\circ}$. The star catalogue includes 2,200 stars and a hyperacuity algorithm performing to $1 / 10$ pixel $(1 \sigma)$. The average number of stars in the image is:

$$
\overline{N_{\text {FOV }}}=\frac{2200 \text { stars }-2200 \text { stars } \cos 11^{\circ}}{2}=20.2 \text { stars }
$$

The PD-error(1б) is then:

$$
\text { PDerror }_{(10)}=\frac{2 \cdot 11^{\circ} \cdot \frac{1}{10} \text { pixel }_{(1 \sigma)}}{650 \text { pixels } \cdot \sqrt{20.2 \text { stars }}}=2.7 \text { arcseconds }
$$

\section{DESIGNING STAR TRACKERS}

In the previous section the accuracy of a star tracker was discussed. The accuracy can be expressed as:

$$
\begin{aligned}
\text { accuracy }= & F(\text { FOV , Star catalogue size , } \\
& \text { CCD chip , Hyperacuity routine })
\end{aligned}
$$

The engineer is faced with the problem of optimizing these parameters toward a star tracker configuration.
Unfortunately the parameters are not independent but rather a trade off between these parameters.

\section{FOV}

The most important parameter is probably the FOV. A little FOV implies an easy design of the camera lens and high accuracy. However, the disadvantage is a very difficult initial acquisition of the attitude (see next paragraph), and the size of the star catalogue is growing to prohibitive sizes, as the FOV decreases.

It is realized that it is difficult to make an optimal choice. The FOV is often determined by external factors. For instance if the CCD camera is shared with a scientific mission, this may constrain the choice of the FOV. Also constraints on the computer memory size may limit how narrow the FOV can be.

\section{Number Of Stars In The Star Catalogue}

It is desirable to have many stars in the image, because it increases the accuracy of the star tracker and because it simplifies the pattern recognition of the star constellations. The stars emit a limited amount of light, and the CCD chip has a limited quantum efficiency. Therefore there are only two parameters left to determine the sensitivity of the system, i.e., the design of the camera lens and the exposure time.

The sensitivity of the camera is primarily determined by the aperture of the front lens. In order to have many stars in the FOV the aperture must be big. The sensitivity is proportional to the area of the front lens. However, as the front lens increases in size, the weight of the lens increases, and the distortion in the lens gets more dominant. This implies that the hyperacuity algorithm performs more poorly, and no accuracy enhancement is achieved.

Increasing the exposure time of the CCD chip is another way to increase the sensitivity of the system. However, the strategy has disadvantages as the update rate decreases and the images will include motion smear. Motion smear tends to decrease the performance of the hyperacuity technique. Therefore the exposure time has to be chosen very carefully as a trade off between many stars and an accurate hyperacuity algorithm. An important aspect in determining the exposure time is whether the spacecraft is 2 or 3 axis stabilised. Also the $\mathrm{S} / \mathrm{N}$ ratio will decrease with increasing exposure time.

\section{The Hyperacuity 'Technique}

The hyperacuity technique has already been described. However there is an enormous dynamic range of illumination from the brightest to the dimmest stars. In the digitalization of the CCD signal there is a limited resolution (typically 7-15 bits). This implies that the brightest stars will overflow and the dimmest stars will be difficult to detect due to noise. The different situations are shown in Fig. 4 (next page).

It is impossible to give an analytic solution to the optimal choice of systems gain/detection level for the stars. It depends on the hyperacuity algorithm and the hardware. 


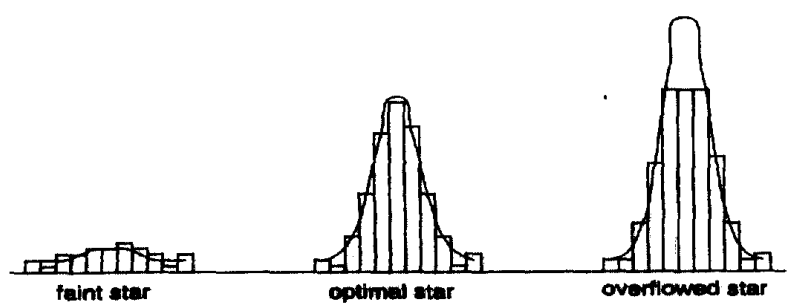

Fig. 4. Different Stars Utilizing Hyperacuity
INITIAL ACQUISITION OF THE ATTITUDE

A major advantage of the second generation star trackers is that no initial attitude from another source is required. The 1st generation of star trackers had to be "locked" on a known star(s) near the center of the image and the star tracker was then able to track this star(s). The advantage of the 2nd generation star trackers is: They are autonomously able to recognize the stars in the image based on the pattern they form. There has been published a variety of algorithms for pattern recognition of star constellations. It is insignificant which algorithm that is actually implemented in the star tracker, as long as the algorithm is robust to missing stars and extra stars. In this paper an algorithm designed by the author is described and tested. Many other algorithms would have performed similarly.

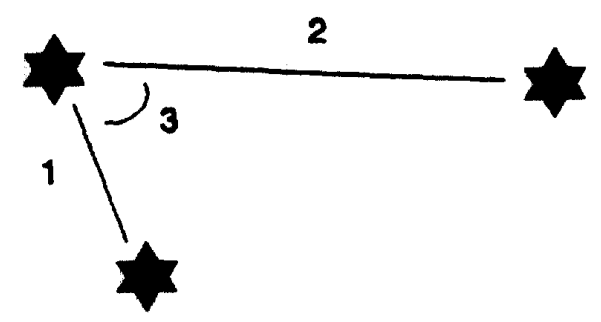

Fig. 5. The Parameters Used to Characterize a Star

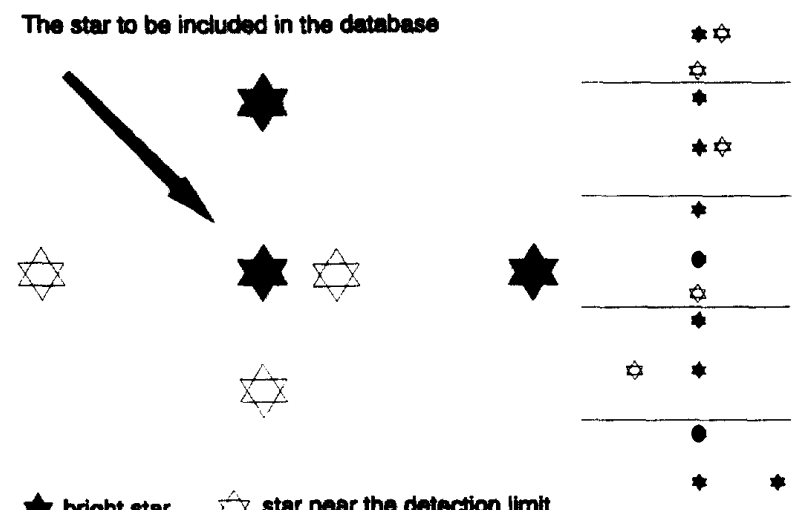

Fig. 6. The Different Constellations Included in the Database Due to the Uncertainty of the Magnitude

\section{Pattern Recognition Of Star Constellations}

A star catalogue is constructed so that there are 20 stars in the FOV on average. For all stars in the sky the angular distance to the first and the second neighbour star is registered. See Fig. 5. A star is then registered by:

1) the angular distance to the first neighbour star;

2) the angular distance to the second neighbour star; and

3) the spherical angle between the two neighbour stars.

Due to the uncertainty on the magnitude some stars near the detection limit are only detected with a certain possibility. Therefore, all possible star constellations are included for all stars, see Fig. 6 . Given a star image, the stars are identified in the database. This typically results in a few candidate positions for each star. However, the correct candidates are easily found comparing the angular distances in the star catalogue and the real image.

Several tests of the algorithms have been performed. In all tests the gain control was adjusted to an average of 20 stars in the FOV. The required database size was calculated as a

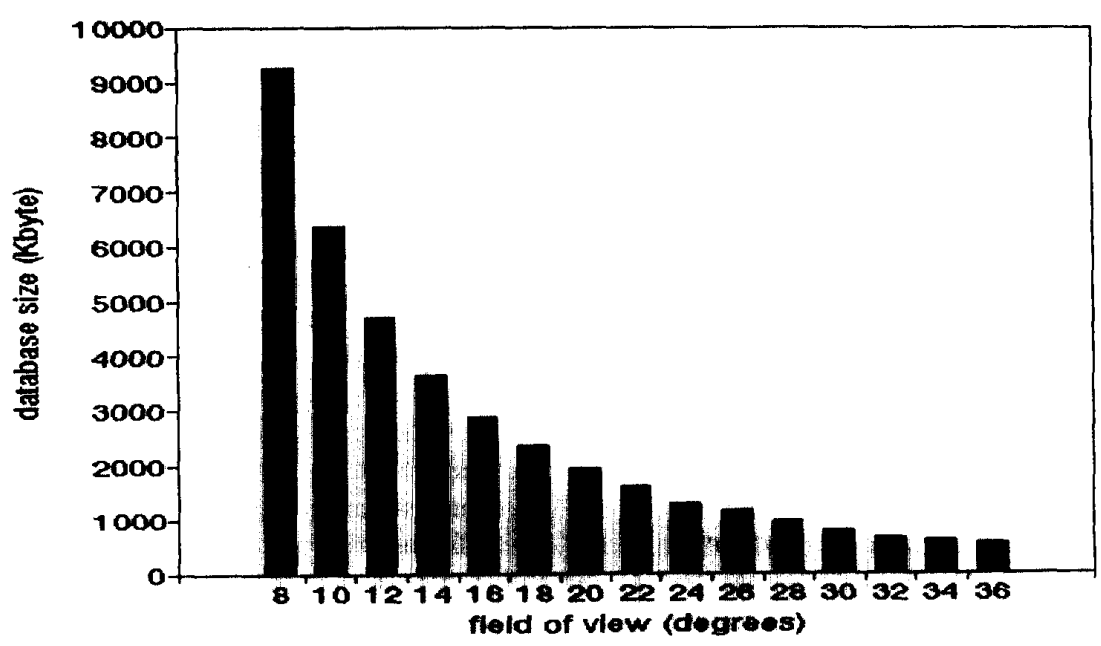

Fig. 7. Size of the Database as a Function of the Field of View 


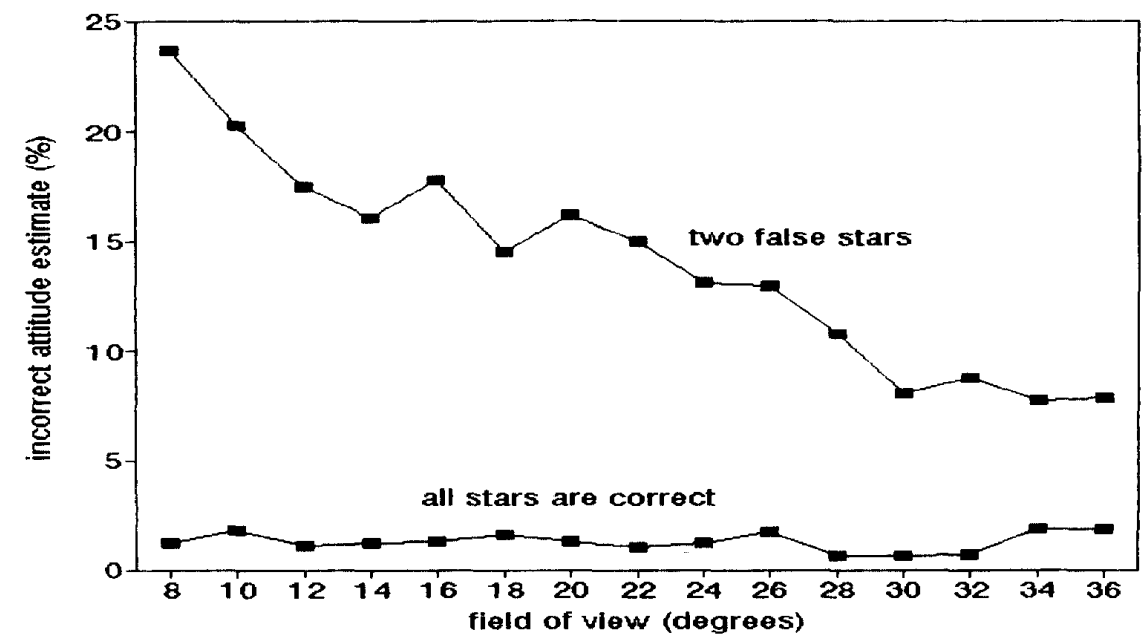

Fig. 8. The Probability of Correct Attitude Estimate as a Function of the FOV. In Two Cases 1) All Stars are Correct; and 2) Two Extra Stars are Added as Noise

\begin{tabular}{||l||l|l||}
\hline \hline Type & $\begin{array}{l}\text { High Precision, } \\
\text { Astronomical }\end{array}$ & Low cost, all purpose \\
\hline \hline Field of view & $\begin{array}{l}\text { Narrow (a couple of } \\
\text { degrees })\end{array}$ & Wide $\left(15^{\circ}-40^{\circ}\right)$ \\
\hline Initial attitude acquisition & No & Yes \\
\hline Power consumption & High $(>20$ Watts $)$ & Low $(<5$ Watts $)$ \\
\hline Weight & High $(>20 \mathrm{Kg})$ & Low $(<4 \mathrm{Kg})$ \\
\hline Accuracy & 1 arcsecond & 20 arcseconds \\
\hline
\end{tabular}

function of the FOV in Fig. 7. Also the probability of a correct initial attitude estimate is shown in Fig. 8 as a function of the FOV in two cases 1) all stars are correct; and 2) two non-existing stars are added as a source of noise.

The conclusion of this investigation is that it is possible to perform a pattern recognition of the star constellations. If the FOV is below $10^{\circ}$ the database size however becomes prohibitively large for most space applications.

The only way to get around this problem is to omit to compile the database and only include the raw star positions. Then all constellations (as shown in Fig. 5) have to be calculated on the spot. The advantage is that no database is required, but the algorithms will execute the order of 1-30 minutes.

\section{TRENISS ON THE COMMERCIAL MARKET}

There are multiple vendors of star trackers on the commercial market. It appears that the market is segmented into two different concepts:
1) Low cost, all purpose star trackers that can be used as a part of the attitude control system in a micro satellite. Typically precision on 20 arcseconds.

2) High precision star trackers capable of determining the attitude better than 1 arcsecond. These are typically used for astronomical missions and are very heavy due to mechanical rigid constructions.

The table above outlines the two types. Especially the low cost, all purpose star trackers are evolving extremely fast. The systems are just beginning to appear, and they are getting lighter and cheaper. No doubt this is an instrument that will appear as standard in all spacecraft in a couple of years.

\section{ACKNOWLEDGMENTS}

The author would like to thank Professor John $\mathbf{L}$. Joergensen for valuable discussions and inspiration throughout this work. The author is also grateful to employees at the Jet Propulsion Laboratory, Guidance and Control Section, for valuable discussions. Part of this work has been sponsored by the Radio Parts Foundation and the Danish Research Academy. 


\section{REFERENCES}

[1] P. Fortescue, J. Stark, Spacecraft Systems Engineering, John Wiley \& Sons Ltd., Sussex, England, 1991.

[2] J.L. Joergensen, A. Damkjaer and C.C. Liebe, A Project Proposal for a Star Imager for Accurate On-line Attitude Determination, Nordic Society for Space Physics, 1991 NSSR Conference Proceedings.

[3] C.C. Liebe, Pattern Recognition of Star Constellation for Spacecraft Appli cations, IEEE Aerospace \& Electronics Sys. Mag., Jan, 1993, pp. 31-39.
[4] R.H. Stanton, J.W. Alexander and E.W. Dennison, CCD Star Tracker Experience - Key results from Astro 1 Flight, SPIE Vol. 1949, Space Guidance, Control and Tracking (1993).

[5] D. Baldini, M. Barni, A. Foggi, G. Benelli and A. Mecocci, A New Star-Constellation Matching Algorithm for Satellite Attitude Determination, ESA Journal 1993, Vol. 17, pp. 185-198.

[6] S. Mossbacher: Flight Hardware Survey, Celestial Sensors, JPL 11/93.

Carl Christian Liebe was born 1966 in Copenhagen, Denmark. He received the M.Sc. degree in electrical engineering at the Technical University of Denmark in 1991 and the Ph.D. degree in image processing at the0 Department of Electrophysics, the Technical University of Denmark in 1994. Currently, Carl Christian Liebe is Assistant Professor at the Technical University of Denmark.

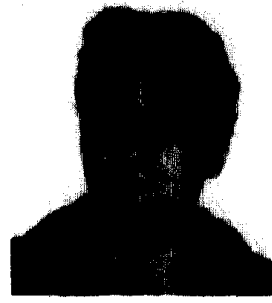

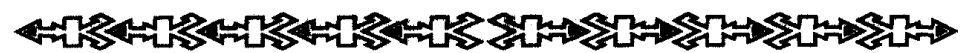

\section{ABSTRACTS}

The following pair of papers were originally submitted for publication in this magazine, but were found to be far too extensive in their coverage - and length - to fit. After consultation with the author, we agreed that the best solution was to publish the Abstracts here and advise readers that the full papers will appear in our Transactions during 1996. If anyone wishes more information prior to publication of full articles, feel free to contact the principle author, John Mulholland, at the Department of Electrical Engineering and Computer Science, Villanova University, Villanova, PA 19085-1681; Phone: (610) 519-7097.

Spacecraft Telemetry Link Performance in a Transfer Orbit by Frank K. Tamney, Jr., Martin Marietta Corp.; and John E. Mulholland, Villanova University.

ABSTRACT. There are several critical periods early in the mission of a geo-stationary communication satellite. The first is the period from launch vehicle ignition until the upper stage final successful burn. The second is after the above span until the vehicle reaches its final altitude of a synchronous orbit. For a nominal low thrust aopgee boost ascent subsystem during that later time, almost continuous telemetry is mandatory. This is especially true during the crucial periods of main engine burns and attitude correction phases.

Maintaining a strong telemetry link throughout this phase requires an adequate radio frequency (RF) signal link from the spacecraft to a ground station in the telemetry RF channel. An analysis of this link performance during each orbit until final position has two major aspects. One, the location of the spacecraft in relation to the ground tracking station at each moment in the mission is a matter of geometry and Keplerian physics. The other is the RF signal and its supporting subsystems, both on the ground and aboard the vehicle. This paper will examine the fundamental theoretical considerations of both the orbit parameters and radio link components and then analyze the individual parameter sensitivities.

Next, a nominal case for a generic mission will be studied. This survey will consider the telemetry performance during each major stage of the flight from the launch through the transfer orbit to the post-injection period to the final orbit. Then abnormal situations due to both orbit and RF faults will be examined. Finally, some design and operation concepts which may lessen the impact of the previous anomalies, are presented.
Intersatellite Laser Crosslinks

by Sean A. Cadogan, Martin Marietta Corp; and John E. Mulholland, Villanova University.
ABSTRACT. Intersatellite laser crosslinks provide a method of communication that has significantly increased the data throughput that can be managed over typical RF communication systems, and has significant growth potential. Optical communications offer very wide bandwidths which can be effectively utilized with wavelength division multiplexing techniques. The data rate growth potential is well beyond the few gigabit per second range of RF technology. The use of lasers in transmitting optical data takes advantage of its small wavelength and low beam divergence to send highly directed signals over significant distances with controlled losses in intensity. The laser's high directivity aids in resistance to jamming communications between satellites, or between satellites and ground stations.

Various intersatellite laser optical crosslink systems are discussed including the Massachusetts Institute of Technology's Laser Intersatellite Transmission Experiment (LITE), and McDonnell Douglas Electronic Systems Company-Laser Crosslink System, and The Ball Aerospace Optical Intersatellite Link, in order to display the various subsystems and their implementations. Link budget calculations are performed on the most commonly used modulation formats to determine system parameters necessary to close the crosslink.

Background is provided on primal system architectures and methods of laser communication, as well as presently implemented systems. The authors provide some insights on where intersatellite laser crosslink systems have opportunity to increase their data throughput and reduce acquisition time. 